\title{
Absorption of calcium from milk and tempeh consumed by postmenopausal Malay women using the dual stable isotope technique
}

\begin{abstract}
Assessment of calcium bioavailability from non-dairy foods containing moderate amounts of calcium is especially important in populations that have habitually low dairy consumption. Absorption of calcium from milk and tempeh (a traditional fermented soy product) was compared in a sample of Malay subjects. A randomized, crossover design was utilized to assess calcium absorption in 20 postmenopausal women from either a glass of milk $(114 \mathrm{~g})$ or from a meal of tempeh (206 g); each containing $130 \mathrm{mg}$ calcium. At each study of Phase 1 (mid-July) and Phase 2 (mid-August), intravenous (42)Ca and oral (44)Ca were administered and calcium absorption was measured in 24-h urine collections post-dosing; with a 1-month washout period between phases. Absorption of calcium from tempeh did not differ significantly from milk (36.9 +/- $10.6 \%$ vs. $34.3+/-8.6 \%$, respectively). Due to differences in the calcium content of tempeh, four servings of this product would be needed to get the same amount of absorbed calcium as that obtained from a 4-ounce glass of milk. Tempeh may provide readily available calcium for this population of women at risk for low bone mass.
\end{abstract}

Keyword: Calcium absorption; Tempeh; Malay; Postmenopausal women; Low bone mass 